\title{
Nos plantamos! Urbanismo participativo y agricultura urbana en los huertos comunitarios de Madrid*
}

\author{
José Luis Fernández de Casadevante
}

\author{
Nerea Morán Alonso**
}

\section{Resumen}

Los huertos comunitarios son una realidad emergente en las ciudades españolas, en Madrid surgen impulsados por asociaciones vecinales, colectivos sociales y ecologistas. Una dinámica que prolifera durante los últimos seis años, construyendo un discurso crítico sobre la sostenibilidad urbana y constituyendo estructuras de coordinación que han conseguido introducir esta temática en la esfera pública y en la agenda política. Estos huertos son espacios que están articulando experiencias de reflexión e intervención participativa sobre la ciudad, sobre el espacio público, las identidades colectivas o los procesos de apropiación espacial. Además desde estas iniciativas se está abordando la noción de soberanía alimentaria como cuestión estratégica para el futuro de las ciudades, promoviendo la incorporación en el planeamiento de la agricultura urbana, como una más de las dimensiones que estructuran la ciudad, aprovechando al máximo su potencialidad no sólo en cuanto a la producción de alimentos, sino en sus aspectos sociales, educativos y ecológicos.

\section{Palabras clave}

Agricultura Urbana; Huertos Comunitarios; Regeneración Urbana; Participación Ciudadana; Urbanismo Participativo

\section{Abstract: We're planting! Participative urbanism and urban agriculture in the community allotments of Madrid}

Community allotments are an emerging reality in Spanish cities; in Madrid they have arisen through the efforts of neighbourhood associations, social groups and ecologists. It is a dynamic that has proliferated over the last six years, building a critical discourse on urban sustainability and forming coordinated structures that have been able to introduce this topic into the public sphere and the political agenda. These allotments are spaces that are articulating experiences of reflection and participative intervention on the city, on public space, group identities and spatial appropriation procedures. These initiatives are also approaching the notion of food sovereignty as a strategic issue for the future of the cities. They are promoting the incorporation of urban agriculture into planning, as another of the dimensions that shape the city, maximizing their potential not only for food production, but also for their social, educational and ecological aspects.

\section{Key words}

Urban Agriculture; Community Allotments; Urban Regeneration; Citizen Participation; Participative Urbanism

\footnotetext{
Recibido: 16/07/2012; aceptado definitivamente: 08/01/2013

"Este texto se basa en gran parte en los resultados del trabajo de investigación "Huertos Comunitarios en Madrid" (inédito), realizado por José Luis Fernández de Casadevante como trabajo final en el Curso de Experto Internacional en Soberanía Alimentaria y Agroecología Emergente de la Universidad Internacional de Andalucía. Tutores: Ángel Calle y David Gallar. Aprobado en mayo de 2012

"Sociólogo, Miembro de Garua S. Coop. Mad. y responsable de Huertos Urbanos de la Federación Regional de Asociaciones Vecinales de Madrid. FRAVM.

***Arquitecta, Personal Investigador en Formación del Dpto. de Urbanística y Ordenación del Territorio, Escuela Técnica Superior de Arquitectura, Universidad Politécnica de Madrid, y miembro del Grupo de investigación en Urbanismo, Arquitectura y Sostenibilidad (GIAU+S, UPM).
} 


\section{Aproximarnos a la huerta de la esquina}

"Un físico es un trozo de materia que investiga la materia. Un biólogo es un trozo de vida que investiga la vida. Un sociólogo es un trozo de sociedad que investiga la sociedad. Todos son espejos que el universo se pone en su centro". Jesús Ibáñez.

La agricultura urbana es una realidad que durante la última década ha ido ganándose un espacio en el marco de los debates estratégicos sobre seguridad alimentaria, sostenibilidad y urbanismo. Esta temática se encuentra presente dentro de la agenda política de instituciones internacionales como la Organización de Naciones Unidas, a través de la Fundación para la Agricultura y la Alimentación (FAO, 2010), evidenciando el papel que juega en la producción de alimentos a nivel planetario y su centralidad a la hora de poner en marcha iniciativas contra el hambre y la pobreza, o encaminadas a la mitigación del impacto del cambio climático.

Actualmente se calcula que hay unos 800 millones de personas dedicadas a la agricultura y ganadería urbanas, que producen entre el 15 y el $20 \%$ de los alimentos del planeta. Se considera que para el año 2020 en las ciudades africanas habrá entre 35 y 40 millones de personas que dependerán de estas actividades para cubrir sus necesidades alimentarias (Karanja y Njenga, 2011). El protagonismo político y académico de la agricultura urbana ha pivotado sobre los países de la periferia, concentrando las iniciativas ciudadanas más ambiciosas, las políticas públicas más innovadoras y la mayor parte de las investigaciones teóricas o aplicadas, como demuestra el trabajo de entidades como la Fundación RUAF ${ }^{1}$.

\footnotetext{
${ }^{1}$ Entidad especializada en la investigación, acompañamiento de las iniciativas de agricultura urbana en países empobrecidos, y una de las principales plataformas de difusión a nivel internacional. <http://www.ruaf.org/node/831> (consultado el 4 de febrero de 2013).
}

Aunque durante la última década vemos un renovado interés en los países enriquecidos por las cuestiones relacionadas con la agricultura urbana. Una preocupación que se traduce en la apertura de líneas de investigación sobre estas temáticas a nivel europeo, como la que plantea el programa de transferencia de ciencia y tecnológica $\mathrm{COST}^{2}$, o los trabajos académicos realizados en Madrid (Verdaguer y Vázquez, 2010) y Cataluña (Arosamena, 2012). Así como en un creciente protagonismo de los movimientos sociales urbanos a la hora de ir incorporando a la esfera pública las cuestiones relacionadas con la horticultura en la ciudad.

En este contexto resulta relevante analizar la experiencia de la Red de Huertos Urbanos Comunitarios de Madrid, como uno de los espacios donde están confluyendo de forma más consistente en nuestra geografía las prácticas y discursos sobre urbanismo participativo, sostenibilidad integral de los ecosistemas urbanos y soberanía alimentaria. La aproximación que hemos realizado a esta experiencia se sustenta en un trabajo de observación participante. Durante un año hemos realizado visitas a distintos huertos, participado en asambleas y reuniones, asistido a jornadas de sensibilización o formación técnica, asesorías a grupos promotores, además de la participación cotidiana desde el huerto de nuestro barrio. Esta tarea reflexiva se ha acompañado de tres entrevistas grupales semiestructuradas con personas de la Red de Huertos Urbanos de Madrid.

\footnotetext{
${ }^{2}$ Ver página del proyecto: <http://www.cost.eu/domains actions/tud/Actions/TD1106> (consultado el 4 de febrero de 2013).
} 


\section{Apuntes para una historia de los huertos comunitarios en Madrid}

Poetas, nunca cantemos la vida de un mismo pueblo, ni la flor de un sólo huerto.

Que sean todo los pueblos y todos los huertos nuestros. León Felipe.

La agricultura siempre ha estado presente en relación con la ciudad, como condición básica para su abastecimiento, hasta que una vez consolidada la revolución industrial, fue desplazada cada vez a mayor distancia debido a la expansión de los mecanismos de transporte y al acceso a energía abundante y barata. El resurgir cíclico de la agricultura urbana ha estado ligado a episodios de crisis (económicas, sociales, conflictos bélicos...) y en la actualidad se puede encontrar bien en proyectos relacionados con la subsistencia (tanto producción destinada a la venta como al autoconsumo) o en una vertiente más comunitaria. Es en esta última tipología en la que nos centraremos, la de los huertos comunitarios, que nacen en los años 70 en las grandes ciudades de Estados Unidos, en un contexto de desindustrialización y de huida de la población con recursos de los centros urbanos, de crisis fiscal, recortes en gastos sociales y pérdida de la calidad de vida de los barrios populares: concentración étnica, elevadas tasas de criminalidad, abandono y proliferación de solares... (Soja, 2006).

La generalizada situación de abandono de muchos barrios desfavorecidos termina generando una dinámica de resistencia, basada en la reorganización comunitaria de los habitantes a partir de la construcción de alianzas de los principales agentes sociales del territorio (asociaciones locales, iglesias, redes informales de apoyo mutuo...). Una reorganización orientada a construir entre los habitantes de dichos barrios comunidades de intereses, logrando visiones compartidas de la realidad y de los problemas sobre los que intervenir, constituyendo nuevas dinámicas y organizaciones sociales que implicaban a las personas afectadas.

Muchas de estas luchas barriales contaron con el apoyo de Organizadores Comunitarios, que cuando eran demandados por las propias comunidades se dedicaban a poner en marcha estos procesos. Entre los organizadores barriales destacan figuras como Saul Alinsky (Hernando, 2006) o Karl Linn, este último trataba de intensificar las relaciones sociales de los habitantes en torno al diseño, construcción y gestión de espacios comunes, que además simultáneamente recuperaban y dignificaban zonas degradadas de estos vecindarios. El diseño implicaba un encuentro de distintas sensibilidades, suponía un ejercicio de reconocimiento de las necesidades y problemas compartidos, y su construcción y gestión constituían la puesta en marcha de un proyecto común.

A estas iniciativas Linn las denominaba comunes vecinales (Linn, 2009), y fueron realizadas por barrios de toda la costa este de EEUU. Estos comunes vecinales eran una palanca que posteriormente permitía abordar otras problemáticas e implementar distintas dinámicas de organización y movilización social. A partir de los años 70 Linn fue uno de los más entusiastas impulsores de los huertos comunitarios y concibió dichos espacios como un ejemplo perfecto de comunes vecinales. El surgimiento de los huertos comunitarios nos remite por tanto al cruce entre las luchas urbanas por la justicia social, emprendidas por las organizaciones comunitarias durante la década anterior, con las movilizaciones y la labor de sensibilización del movimiento ecologista sobre estilos de vida más sostenibles. Muchas de estas experiencias arrancan con la ocupación de solares y espacios abandonados reconvertidos en huertos 
que son utilizados como herramienta de apoyo comunitario que relaciona la calidad ambiental, la cohesión social y la educación (Lawson, 2005). Una dinámica que a lo largo de la década de los 70 se replica en la mayoría de los países europeos, llegando a nuestra geografía con cerca de tres décadas de retraso por múltiples motivos (dictadura, industralización tardía...).

¿Qué crisis atraviesan actualmente nuestras ciudades para que emerjan estas temáticas y dinámicas organizativas? Seguramente no exista una crisis en singular, sino la superposición de diversas crisis que se expresan en una dinámica bautizada como cambio global: pico del petróleo, crisis socioeconómica, ecológica, alimentaria, de representación política y del modelo urbano (VVAA, 2009). Un cambio que aumenta la creciente conciencia de encontrarnos ante un periodo de transición, en el que los huertos comunitarios emergen como espacios donde arraigar un malestar social difuso y construir dinámicas alternativas.

Desde hace unos años en Madrid, asociaciones vecinales, colectivos juveniles, comunidades educativas y grupos ecologistas han comenzado a convertir en huertos comunitarios múltiples espacios abandonados, deteriorados o que debido a su emplazamiento se encontraban infrautilizados (solares, parcelas tapiadas durante lustros, espacios dentro de parques de gran tamaño, zonas baldías bordeando autopistas y vías férreas...).

Las comunidades locales que dinamizan huertos comunitarios se organizan para restaurar a pequeña escala espacios urbanos degradados, conjugando la devolución del valor de uso a espacios urbanos abandonados, con una "rehabilitación relacional" (Fernandez de Casadevante y Ramos, 2010) que busca restablecer la calidad de los espacios mediante la intensificación de las relaciones sociales (desarrollando actividades como fiestas populares, actividades educativas 0 iniciativas culturales).

La primera iniciativa surge en 2004 de la mano de la asociación GRAMA (Grupo de Acción para el Medio Ambiente), que consigue que la asociación que gestiona el albergue juvenil de la Casa de Campo, les ceda un terreno colindante. Una vez preparado el terreno se pone en marcha el huerto. Desde el primer año la tierra ha dado cosechas que mejoraban según aumentaba la destreza de los hortelanos y se iba aumentando la fertilidad del suelo. El proyecto, más allá de disponer de un huerto, persigue la puesta en marcha de una modesta escuela de hortelanos donde formar a gente interesada en aprender a cultivar de forma ecológica. Desde entonces el huerto continúa y se han realizado multitud de cursos y de actividades de sensibilización.

Hay que esperar al año 2006 para que veamos surgir el primer huerto comunitario en nuestra ciudad, en la Plaza de Corcubión del barrio de El Pilar. En las jornadas previas a la llegada del verano un grupo de vecinos, vinculados al movimiento vecinal y asociativo del barrio, comienzan las labores de limpieza y acondicionamiento de un espacio degradado, sucio y en desuso, para convertirlo en un huerto comunitario. Como sus impulsores afirman: "la ilusión y el empeño por crear espacios públicos y participativos en el barrio, que mejoren el tejido social y favorezcan la calidad de vida, se ha conseguido plasmar en este huerto comunitario" ${ }^{3}$. Desde entonces este espacio se ha consolidado como un referente en el barrio que ha permitido el encuentro vecinal, la relación entre personas que cohabitando en un

\footnotetext{
${ }^{3}$ Ver:< http://www.lapiluka.org/el-huerto-del-barrio/> (consultado el 4 de febrero de 2013).
} 
bloque no sabían cómo se llamaban sus vecinos. Además de suponer la mejora ambiental y el embellecimiento del espacio el huerto se ha convertido en un dinamizador de la convivencia, y en un espacio polivalente donde sembrar y cuidar plantas, realizar actividades culturales, conversar o realizar una comida popular. $Y$ es que como dicen "este huerto da algo más que hortalizas". Una iniciativa modesta que sin embargo es muy conocida fuera del barrio, ya que debido a su carácter pionero se convierte en referente y estímulo para muchas de las experiencias que posteriormente se pondrán en marcha en la ciudad.

El goteo de experiencias continúa con la iniciativa de Esta es una plaza ${ }^{4}$, que en el marco de unas jornadas de acciones urbanas realizadas en $\mathrm{La}$ Casa Encendida a finales de 2008, consigue una cesión temporal para reconvertir un solar abandonado durante más de 30 años, en una suerte de plaza pública en el barrio de Lavapiés. Esta ágora de barrio con teatro y espacio para actividades culturales, zona deportiva y huerto, donde todo es autoconstruido, busca fórmulas para conseguir su continuidad cuando acaba el permiso concedido para la realización de las jornadas y se clausura nuevamente el espacio. Finalmente se decide constituir una asociación (Esta es una plaza) y presentar al Ayuntamiento un proyecto de Plan de Autogestión Vecinal del solar, que se va negociando a la vez que se realizan algunas actividades para mantener viva la iniciativa y legitimarla públicamente, como son los Desayunos Públicos frente a su entrada. Tras varios meses de conversaciones, en mayo de 2009 todo lo que se había construido en el solar es arrasado por excavadoras municipales. El malestar vecinal y las acciones de denuncia que se realizan como

\footnotetext{
${ }^{4}$ Ver: <http://estaesunaplaza.blogspot.com.es/> (consultado el 4 de febrero de 2013).
}

respuesta tienen sus frutos, ya que en el mes de junio el Ayuntamiento concede un permiso temporal para usar el solar (cesión en precario ${ }^{5}$ ). Desde entonces la iniciativa no ha parado de profundizar en su dimensión participativa y de crecer conformando un verdadero espacio cultural multiuso en el antiguo solar, en el que nuevamente ha habido espacio para la construcción de un huerto comunitario.

Y llega el año 2010 en el que podríamos fechar la emergencia de las experiencias de huertos comunitarios, ya que coincide de forma sincrónica pero no coordinada la puesta en marcha de varios huertos comunitarios por parte de asociaciones vecinales. Esta proliferación da pie a la primera estructura de coordinación en la Federación Regional de Asociaciones Vecinales - FRAVM, como un espacio de encuentro, intercambio de experiencias, recursos y creación de mecanismos de apoyo mutuo. Además de ofrecerse como punto de orientación para las personas y entidades interesadas en poner en marcha iniciativas similares en sus barrios y municipios, $y$, por último, iniciar un trabajo de intermediación con la administración en la búsqueda de modelos de regularización y cesión de espacios. Actualmente desde las entidades de la FRAVM se gestionan 18 huertos y se mantienen relaciones de acompañamiento con otros 8 grupos promotores.

Meses después de este proceso, nace la Red de Huertos Urbanos Comunitarios de Madrid (REHD $M A D)$, donde además de las experiencias impulsadas por las entidades vecinales, confluyen

\footnotetext{
${ }^{5}$ La cesión está condicionada al requerimiento del solar por parte del Ayuntamiento, en el momento en que éste lo reclame para desarrollar la dotación cultural prevista en este espacio, la asociación deberá abandonarlo, sin derecho a solicitar ninguna compensación y con la obligación de dejarlo en las mismas condiciones en que lo encontró. Además en el contrato se establece que la asociación asumirá los gastos y procedimientos derivados de la realización de obras de mejora en el espacio, sin poder recurrir a los medios de la administración local en este sentido.
} 
otras iniciativas hortícolas de la ciudad vinculadas a entidades ecologistas, colectivos vecinales 0 comunidades educativas de distintos campus universitarios. La Red es una apuesta por coordinar al conjunto de proyectos de huertos comunitarios de la ciudad, de cara a dotar de una mayor visibilidad al conjunto de las iniciativas ${ }^{6}$, fomentar el intercambio de experiencias (visitas, encuentros...), compartir recursos (semillero, intercambio de semillas, compras colectivas de estiércol...), así como crear mecanismos de apoyo mutuo, y promover espacios formativos (jornadas, seminarios, cursos...), además de ofrecer un espacio de referencia, desde el que prestar asesoría y orientación a las personas y entidades interesadas en poner en marcha huertos similares. Un trabajo que contempla realización de manuales temáticos (compostaje, riego, rotaciones y asociaciones de plantas, autoconstrucción de mobiliario con palés...) o protocolos de actuación para poner en marcha nuevas iniciativas.

El número de proyectos que aglutina la Red ronda la treintena, además de mantener relación con otras iniciativas impulsadas de la mano de Asambleas Populares del Movimiento 15-M, algunas de las cuales confluyen en su desarrollo con asociaciones vecinales. La mayor parte de estas experiencias se concentran en los barrios populares del centro y en las antiguas periferias obreras donde mayor presencia tiene el movimiento asociativo. Las zonas urbanas donde se ubican son generalmente espacios pendientes de desarrollo urbanístico (zonas de equipamiento y alguna destinada a uso residencial), así como espacios verdes degradados o zonas interbloque.

En mayo de 2012 se realiza el primer gran encuentro de la Red, una jornada destinada tanto a

\footnotetext{
${ }^{6}$ Ver: <http://redhuertosurbanosmadrid.wordpress.com/> (consultado el 4 de febrero de 2013).
}

facilitar que se conocieran los hortelanos de los distintos barrios, como a dar a conocer este movimiento emergente de horticultura urbana. Un espacio en el que se organizaron distintos talleres (horticultura, autoconstrucción de mobiliario con pallets, huerto educativo y recursos compartidos de la Red), se realizó un pasacalles, una comida popular, conciertos y una charla sobre la historia de la agricultura urbana en Madrid.

Un primer análisis de la evolución de estas iniciativas en Madrid, relativamente consolidadas en la actualidad, nos permite mostrar sus principales características:

- Son espacios públicos autoconstruidos (diseñados, construidos, mantenidos...) y abiertos a la participación ciudadana mediante dinámicas autogestionarias (organizados participativamente por sus usuarios, decidiendo colectivamente los mecanismos de funcionamiento, la planificación del espacio y los cultivos, así como el reparto de las tareas de cuidado y mantenimiento).

- Son espacios verdes sostenibles, ya que funcionan bajo los principios de la agroecología (optimizar espacios, policultivos, variedades locales, insumos y control de plagas ecológicos, compostaje comunitario...).

- Los huertos comunitarios son gratuitos, no se cobra por participar, aunque colectivamente se aborde la dimensión económica y los mecanismos de financiación de cada iniciativa.

- Acceso a tierras cultivables en espacios urbanísticamente consolidados, que se gestionan de forma colectiva y en los que generalmente no hay asignación individual de parcelas o bancales, aunque en dos de las experiencias más recientes el espacio se distribuye entre los hortelanos, cediendo parcelas de $30 \mathrm{~m} 2$ a cada familia adjudicataria. 
La producción se reparte entre la gente que trabaja el huerto, se destina a comidas populares o se regala, pero no está destinada a la venta.

- Son espacios inclusivos que sirven de herramienta para la extensión, coordinación y renovación de los tejidos asociativos, articulando redes formales (AMPAS, grupos scouts, colectivos juveniles, Centros de Día o de terapia ocupacional...) e informales de los barrios.

Las parcelas no son muy amplias, su superficie oscila entre los $1000 \mathrm{~m}^{2}$ de las experiencias más solventes y con terrenos más amplios, a los más pequeños con unos $100 \mathrm{~m}^{2}$, con un carácter más testimonial. Los espacios en los que se asientan son parcelas no agrícolas, con suelos muy compactados y de mala calidad, que son mejorados mediante diversas estrategias (vaciados, construcción de bancales elevados, aporte de insumos ecológicos...).

El volumen de personas que participa ronda las 50 por iniciativa, contemplando distintos grados de implicación que van desde los simpatizantes, a quienes se pasan puntualmente y quienes dinamizan las experiencias. Los grupos motores 0 dinamizadores suelen ser de unas 15 personas, un colectivo que se va ampliando en la medida en que las exigencias de participación son más modestas. Todas las iniciativas se organizan utilizando metodologías participativas y desarrollan de forma periódica jornadas colectivas de trabajo (Huertas Abiertas, Domingos Verdes, Hacenderas...).

Los perfiles de los dinamizadores de los huertos comunitarios generalmente se encuentran entre los 30 y los 40 años, predominando las personas con formación universitaria y empleo estable. Entre las personas que conforman las iniciativas se da una amplia diversidad social de edades, perfiles biográficos y procedencias, salvo el perfil de la población de origen migrante que se encuentra débilmente incorporado.

Los huertos comunitarios han brotado en los márgenes de la ciudad gracias al impulso ciudadano, haciendo frente a las incertidumbres derivadas de la situación de alegalidad y a dificultades como el acceso al agua. El tema salta a la esfera mediática una vez que se ha generando una masa crítica de experiencias suficiente, se han consolidado los ejercicios de coordinación y se han establecido alianzas con la universidad. La simpatía y buena acogida de los huertos comunitarios ha permitido que entren en la agenda política. El Ayuntamiento de Madrid ha empezado a poner en marcha algunas políticas públicas como los huertos de Madrid Salud, que son experiencias mixtas de huertos terapéuticos y vecinales, o el Centro de Educación Ambiental Huerto del Retiro, especializado en temas de horticultura urbana y que forma parte de la Red de Huertos Comunitarios. Desde hace más de un año y medio se mantienen conversaciones entre la FRAVM y el Ayuntamiento de cara a buscar una fórmula que regule y proteja los huertos comunitarios. Una propuesta que dé seguridad jurídica al ayuntamiento a la vez que respete la autonomía de las iniciativas ciudadanas.

Siguiendo la estrategia de visibilizar y construir legitimidad social en torno a los proyectos de huertos comunitarios, la Red de Huertos Urbanos Comunitarios de Madrid ha sido reconocida por el VI Catálogo Español, del Ministerio de Fomento, como Buena Práctica en sostenibilidad urbana. Un reconocimiento a esta iniciativa de la sociedad civil que también ha concedido la ONU, a través del Concurso Internacional HABITAT. 


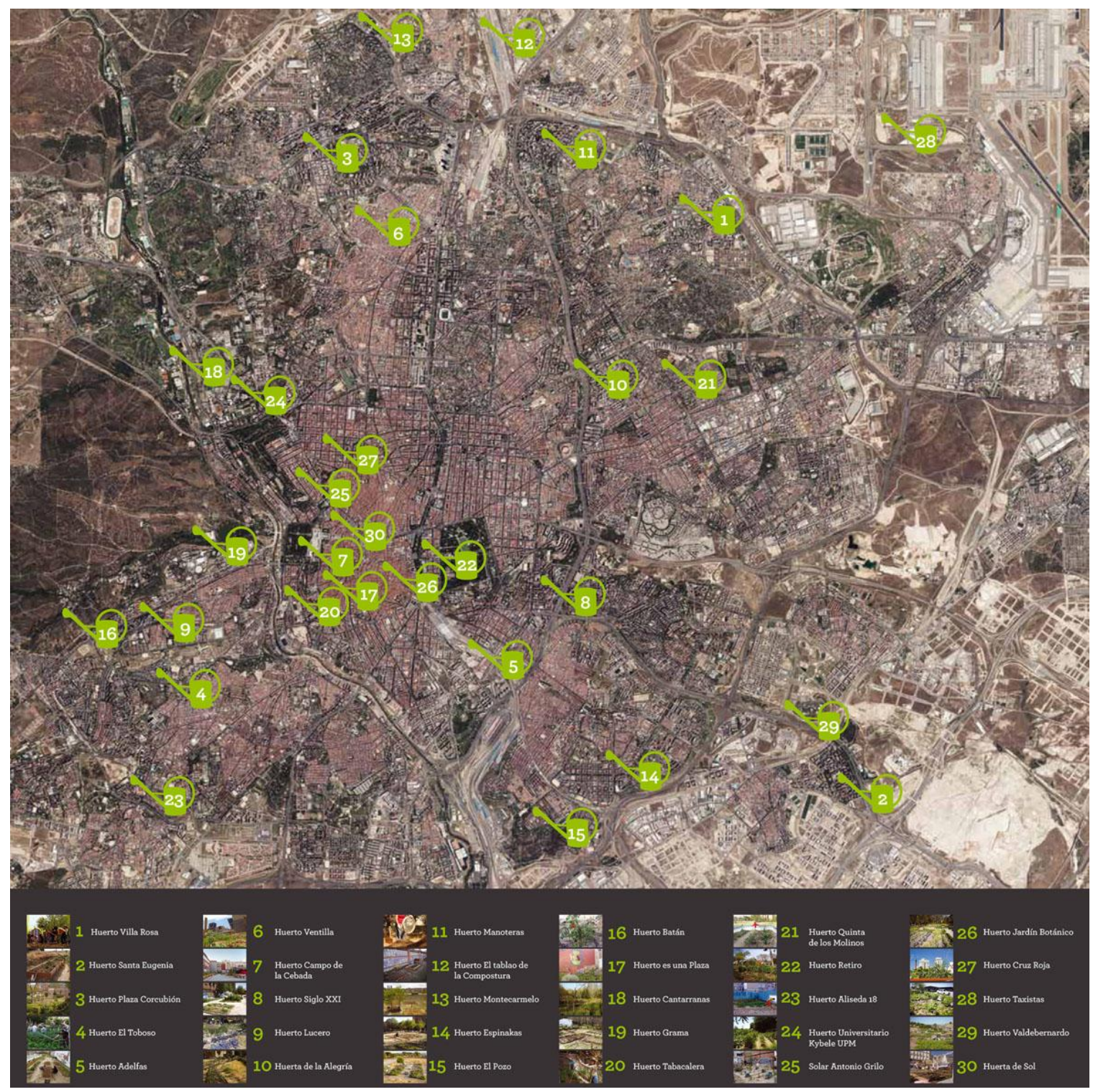

Figura 1. Mapa Huertos Urbanos de Madrid (Todos son comunitarios excepto 26, 27, 28, 29 y 30). Imagen extraída de la exposición Plantando Redes: Huertos urbanos en Madridi. La Casa Encendida.

\section{Urbanismo participativo y horticultura comunitaria: producir relaciones sociales y alimentar modelos alternativos de ciudad}

"Los movimientos sociales urbanos como agentes de unas políticas marcadamente espaciales en la ciudad, al focalizar el espacio urbano como el punto de conflicto y valerse del espacio como recurso para la movilización política, politizan los espacios urbanos en términos de propiedad, usos y significados". F. Tonkiss.

Históricamente las grandes transformaciones urbanas han ido asociadas a proyectos orientados a reformar drásticamente determinadas zonas, mediante la implantación de infraestructuras de transporte o comerciales sobre el trazado de la ciudad consolidada o el desarrollo de extensos procesos de renovación. Una cirugía urbana agresiva, costosa y que pone el énfasis en los cambios materiales (arquitectónicos o urbanísticos) para lograr las mejoras a las que aspiraban los planificadores.

Ante este paradigma ha ido emergiendo durante las últimas décadas una nueva mirada sobre las dinámicas de transformación urbana que incide en la forma en que pequeñas y sutiles iniciativas pueden tener una amplia capacidad de incidencia socio-urbanística. Experiencias localizadas, impulsadas con escaso presupuesto y que resaltan 
los cambios en la dimensión relacional entre las personas y de estas con el entorno. El arquitecto brasileño Jaime Lerner, ex alcalde de Curitiba, las ha defendido como Acupuntura Urbana ${ }^{7}$, otros autores han huido de las metáforas médicas pero mantienen una coherencia con estas reflexiones, cuando hablan de la necesidad de Reparar las ciudades $^{8}$ mediante la puesta en marcha de iniciativas locales que permitan reconstruir la dimensión comunitaria de los vecindarios, la reapropiación del entorno urbano y la preocupación por el medio ambiente, desarrollando transformaciones participativas de espacios urbanos concretos. Una lógica emergente que viene a plantear que la transformación de las ciudades en clave de sostenibilidad y justicia social debería enfatizar cambios en los estilos de vida, las percepciones y expectativas sociales, o los usos del espacio. Desplazando la centralidad que en el discurso urbanístico ha tenido la intervención sobre el patrimonio construido, sin obviar las enormes posibilidades de mejora existentes en términos de rehabilitación física, pero incidiendo en los aspectos sociales y participativos de una regeneración urbana integral ${ }^{9}$.

Los huertos comunitarios se situarían en esta línea de transformación de lo urbano desde lo

\footnotetext{
7"Siempre tuve la ilusión y la esperanza de que con un pinchazo de aguja sería posible curar las enfermedades. El principio de recuperar la energía de un punto enfermo o cansado por medio de un simple pinchazo tiene que ver con la revitalización de ese punto y del área que hay a su alrededor. Creo que podemos y debemos aplicar algunas "magias" de la medicina a las ciudades, pues muchas están enfermas, algunas casi en estado terminal. Del mismo modo en que la medicina necesita la interacción entre el médico y el paciente, en el urbanismo también es necesario hacer que la ciudad reaccione. Tocar un área de tal modo que pueda ayudar a curar, mejorar, crear reacciones positivas y en cadena. Es necesario intervenir para revitalizar, hacer que el organismo trabaje de otro modo." (Lerner, 2003)

${ }^{8}$ Ver el proyecto City Repair Project del arquitecto Mark Lakerman: <http://cityrepair.org/> (consultado el 4 de febrero de 2013)

${ }^{9} \mathrm{El}$ enfoque de la regeneración urbana integral está adquiriendo importancia en el estado español, en el ámbito académico se puede destacar la línea de investigación desarrollada por el Departamento de Urbanística y Ordenación del Territorio de la Escuela Técnica Superior de Arquitectura de Madrid (<http://www2.aq.upm.es/Departamentos/Urbanismo/blogs/rehab/>, consultado el 4 de febrero de 2013)
}

humano, planteando nuevas problemáticas, que no estaban previstas ni eran contempladas por los planificadores o los gestores de la ciudad. Ejercicios de microurbanismo que al implicar a los habitantes en la transformación material de su entorno facilitan la apropiación espacial por parte de los ciudadanos, la redefinición de identidades colectivas a nivel local y una percepción más positiva del territorio, mediante la recuperación activa para el cultivo de lugares degradados.

Resulta destacable como, mediante esta participación en los proyectos, se acaba poniendo a disposición de las iniciativas recursos (depósitos de agua, materiales, conocimientos técnicos...) y redes personales, recreando un lazo social que, más allá de los tejidos asociativos, densifica las redes informales de apoyo mutuo (cuidados en enfermedades, clases de ordenadores a personas mayores, compartir vivienda...).

"Yo llegué nueva a este barrio y para mi ha sido integrador en la vida del barrio, me abrió la puerta a todo lo demás. Mi primer contacto con el centro social y el barrio fue a través del cartel del colegio de Estamos montando un huerto. Eso me hizo llegar luego al grupo de consumo, al centro social, a conocer gente que ahora son muy importantes en mi vida que tienen que ver con esto" 10

"Los huertos los concibo como escenarios de transformación, controlas que ingredientes entran pero el resultado que se va a generar es sorpresa, como en todo lo participativo. Sabes con qué cuentas al entrar pero el resultado es incógnito. Me parece que además de acupuntura urbana, de esponjar el tejido, el papel que juegan es a nivel humano de socializar o resocializar las relaciones y luego volver a conformar comunidades"11

El espacio público tiene un valor relacional, y como tal se encuentra definido por su uso colectivo y su multifuncionalidad. La calidad del mismo

\footnotetext{
${ }^{10}$ Entrevista realizada por los autores a miembros Red de Huertos.

${ }^{11}$ lbíd.
} 
se debería evaluar "por la intensidad y calidad de las relaciones sociales que facilita, por su capacidad para generar mixtura de grupos y comportamientos, por su cualidad de estimular la identificación simbólica, la expresión y la integración cultural" (Borja, 2003). Estos valores y cualidades que caracterizan al espacio público son los que paulatinamente van sufriendo un lento proceso de erosión, debido a los estilos de vida, las políticas, los diseños urbanos y arquitectónicos dominantes.

\begin{tabular}{|l|l|}
\hline Espacio público convencional & Huertos comunitarios \\
\hline Limitación de los usos a aquellos previstos. & $\begin{array}{l}\text { Polivalentes y permiten los usos emergentes no diseña- } \\
\text { dos. }\end{array}$ \\
\hline $\begin{array}{l}\text { Gestión delegada en lo privado o de las Administraciones } \\
\text { Públicas. }\end{array}$ & Gestión Comunitaria y corresponsable. \\
\hline Espacio dado y acabado. & Espacio autoconstruido. \\
\hline Diseño técnico (arquitectura, urbanismo...). & $\begin{array}{l}\text { Diseño autorreflexivo, incorporando multiplicidad de } \\
\text { saberes, técnicos y experienciales. }\end{array}$ \\
\hline Progresivamente mercantilizado. & Desmonetarizado. \\
\hline Espacio que produce distancia o extrañamiento. & Espacio apropiado por los habitantes. \\
\hline Ciudadanía tiene rol de usuario & Ciudadanía productora/gestora/usuaria. \\
\hline $\begin{array}{l}\text { Concebidos de forma seriada y desterritorializada (infra- } \\
\text { estructura, morfología, especies cultivadas...). }\end{array}$ & $\begin{array}{l}\text { Adaptados en mayor medida a la particularidades del } \\
\text { territorio (infraestructura, morfología, especies cultiva- } \\
\text { das...). }\end{array}$ \\
\hline Construido mayoritariamente con materiales nuevos. & $\begin{array}{l}\text { Construidos mayoritariamente con materiales reciclados } \\
\text { o recuperados. }\end{array}$ \\
\hline
\end{tabular}

Tabla1. Características del espacio público convencional (erosionado) frente al espacio público de los huertos comunitarios. Elaboración propia.

La ciudad que maximiza los espacios funcionales para la circulación de personas y mercancías, minimiza la generación de espacios orientados a intensificar las relaciones sociales. Esta pérdida del protagonismo de los espacios públicos coincide con una progresiva pérdida de influencia de las comunidades locales en los debates y espacios de decisión en cuestiones que les afectan. Los huertos comunitarios deben ser entendidos como mecanismos que tratan de revertir estas dinámicas, mediante la promoción de espacios públicos autoconstruidos para el encuentro, la expresión y la producción de procesos comunitarios.

"Interesa como espacio público pero como espacio que no viene dado, un espacio cero donde entra en juego la creatividad de las personas implicadas en el proyecto. Espacios públicos que no existen en la ciudad y los construimos entre las experiencias de los vecinos y ciudadanos. Me interesan como lugar de encuentro, más que como lugar donde experimentar con hortalizas y plantas, más como sociali- zación y conocimiento colectivo"12

Los huertos comunitarios funcionan como un contrapeso, una suerte de centros sociales al aire libre, de forma que generan espacios polivalentes (educativos, culturales, expresivos, convivenciales, hortícolas...), articulan localmente una pluralidad de sensibilidades, demandas y reivindicaciones (ambientales, vecinales, políticas, relacionales...). Espacios inclusivos a la diversidad social, donde se están dando inéditos acercamientos intergeneracionales entre jóvenes y mayores, entre personas de procedencias y formaciones muy heterogéneas.

"Lo que más me emociona es lo que aporta al barrio, porque cosecha no es lo principal que buscamos. Viene gente tan dispar chavales, viejos, gente sola, grupos... Y me mola mogollón porque la gente dice que pasa habitualmente, que están pendientes, que siempre vienen, me parece que es hacer barrio mogollón" 13

\footnotetext{
${ }^{12}$ lbíd.

${ }^{13}$ lbíd.
} 
El proceso de recuperar un espacio degradado, proyectar su diseño hacia el futuro, construirlo y mantenerlo mediante la acción colectiva es lo que dota a los huertos comunitarios de una inigualable capacidad de apropiación por parte de la ciudadanía. La apropiación es el proceso por el que un espacio deviene para individuos y grupos un lugar "propio", la forma mediante la cual establecen vínculos con el lugar, de forma que las percepciones y las acciones que se desarrollan en él resultan apropiadas, correctas (Vidal y Pol, 2005).

"Apropiarse de un lugar no es sólo hacer de él una utilización reconocida sino establecer una relación con él, integrarlo en las propias vivencias, enraizarse, y dejar la propia impronta, organizarlo y devenir actor de su transformación". (Chombart de Lauwe, 1976).

El heterodoxo pensador Ivan Illich no se cansaba de afirmar que "el desarrollo económico ha cubierto de cemento el mundo habitable. El medio ambiente se ha vuelto tan duro que nuestros cuerpos ya no pueden marcar en él su impronta. Así, "pasamos por la vida sin dejar huella" (Illich, 1983). Los huertos comunitarios vuelven a posibilitar que dejemos una huella de nuestra vida en el paisaje urbano, reafirmando e intensificando nuestra relación con el espacio local, desarrollando lo que algunos autores han denominado como topofilia (Tuan, 2007). Una experiencia difícilmente comparable a la que se vive de forma cotidiana mediante los usos que se hacen de los espacios públicos convencionales.

El valor añadido de las experiencias de urbanismo participativo se sustenta en la incorporación de una mayor diversidad social a la hora de realizar diagnósticos, diseñar propuestas e implementar soluciones. Iniciativas que prestan una especial atención a los grupos sociales más infrarepresentados (inmigrantes, mujeres, jóvenes, mayores...) y a los discursos emergentes, inno- vadores, que aún siendo minoritarios encierran las mayores potencialidades de transformación social mediante el desborde de lo previsible y lo esperado (Villasante, 2006).

Desde el urbanismo participativo se ponen en juego una mayor diversidad de miradas sobre la realidad, por lo que se generan diagnósticos más complejos, se construyen consensos compartidos por redes sociales más extensas y se articulan iniciativas más inclusivas y autosostenibles. Un dialogo entre saberes técnicos y profanos, ajenos al territorio y convivenciales, teóricos y prácticos, institucionales y no institucionales, que nos remiten a una forma más abierta de concebir y construir la ciudad.

Los huertos comunitarios también articulan en torno a sus proyectos una amplia pluralidad de conocimientos y saberes sobre lo urbano. Al ser iniciativas autoorganizadas deben realizar una gestión del conocimiento que combine su dimensión democrática, con el aprovechamiento de los saberes y competencias técnicas presentes entre las personas que dinamizan los huertos. Resulta interesante valorar cómo estas iniciativas permiten aplicar las competencias técnicas más diversas (urbanismo, legislación, sociología, educación, arte, agricultura, ecología...). La actividad política y el trabajo hortícola suponen una salida a intereses profesionales y saberes especializados de una generación sobrecualificada que el mercado infravalora o ignora. Corpus teóricos y metodológicos que resultan secundarios en las universidades de arquitectura, urbanismo, sociología o agronomía, se activan en la práctica política y esto lleva a que muchas personas tengan "experiencias profesionales autorreflexivas, se planteen el problema de cómo enriquecer la relación con su profesión desde una proyección territorial [...] reforzando directamente los lugares constructivos de comunidades locales" (Magnaghi, 2012).

Entre los saberes que se están poniendo en valor 
desde los huertos comunitarios encontraríamos los conocimientos campesinos y agrícolas, despreciados tradicionalmente desde los entornos urbanos. Además de ofrecer problemáticas concretas (agronómicas, experienciales, políticas, ecológicas...) donde dialogan conocimientos populares y científicos, estas dinámicas de producción y gestión de conocimiento se vertebran a partir del manejo de las metodologías participativas en las que se basa el funcionamiento cotidiano de las iniciativas.

La morfología de nuestras ciudades, la dificultad para acceder a tierras cultivables o los estilos de vida urbanos, implican que la noción de huerto urbano se diferencie mucho del imaginario que transmiten las huertas tradicionales. Los espacios, los formatos, las imágenes que asociamos a la agricultura urbana son mucho más plurales y diversos que la parcela de tierra con surcos. La pluralidad de localizaciones y casuísticas espaciales, de motivaciones por las que la gente se acerca a participar (inquietudes ambientales o sociopolíticas, ganas de conocer gente, de recuperar espacios degradados, de aprender horticultura...), de grupos sociales que los promueven (comunidades educativas, asociaciones vecinales, colectivos juveniles, grupos ecologistas, de personas con diversidad funcional...), hacen que el rasgo más característico de estas iniciativas de agricultura urbana sea la hortodiversidad. Cada huerto comunitario viene a ser un reflejo de cada comunidad humana que lo impulsa y cuida, lo que implica que cada uno sea radicalmente diferente.

"Igual que la biodiversidad es el salvavidas de la vida, la hortodiversidad va a ser el salvavidas de los huertos urbanos, que la gente vea que hay más modelos de huertos que en el que tú has estado. $Y$ que hay tantas formas de llegar, hay tantas formas de estar, tantas formas de concebirlos, es pedagógico y es educativo que no todos los huertos sean réplicas iguales, que los haya en terrenos ganados a la administración, que los haya en terrenos públi- cos, que los haya en terrenos ocupados, que los haya con una asociación de vecinos pero que los haya en una asociación de artistas, que los haya juveniles pero que los haya en geriátricos. Me parece que esa es su fortaleza" 14

La diversidad de huertos urbanos, en cuanto a sus objetivos, su modo de organización, su diseño, las actividades que se practican en ellos, etc. supone un modo de intervención en la ciudad que se podría incluir en lo que Christopher Alexander denomina procesos de crecimiento a pequeñas dosis, en los que las transformaciones en el entorno se realizan lentamente, a pequeños pasos, desde el conocimiento profundo del espacio local, pero sin aislarse en él, sino pensándolo hacia fuera, de modo que contribuya a mejorar el conjunto de la ciudad. "La diferencia básica entre los dos tipos de crecimiento puede definirse, en términos filosóficos, del siguiente modo: El crecimiento a grandes dosis depende de una visión discontinua y estática del medio ambiente humano; el crecimiento a pequeñas dosis depende de una visión dinámica y continua del medio ambiente. [...] El crecimiento a grandes dosis se basa en la idea de reemplazamiento. El crecimiento a pequeñas dosis se basa en la idea de reparación." (Alexander, 1976). El crecimiento lento permite desplegar multitud de soluciones que dependen de los diversos espacios y comunidades locales, y aprender de los pequeños aciertos y errores, en un proceso de continuo aprendizaje y adaptación. Este modo de actuación es un ejemplo claro de estrategias de resiliencia en un entorno urbano, basadas en la adaptación, la diversidad, la autoorganización y la capacidad de aprender e innovar a partir de la propia historia $^{15}$.

\footnotetext{
${ }^{14}$ Entrevista realizada por los autores a miembros Red de Huertos.

${ }^{15}$ Sobre el concepto de resiliencia y socioecosistemas adaptativos ver los trabajos del Stockholm Resilience Centre, dirigido por el profesor Carl Folke, centro de investigación transdisciplinar que desarrolla distintas líneas de investigación sobre
} 
La recuperación participativa de lugares abandonados o degradados para desarrollar huertos comunitarios esboza algunas tendencias de lo que debería ser un urbanismo emergente. Una forma de pensar el espacio público orientada al reciclaje de suelos abandonados para devolverles un valor de uso, al desarrollo de entornos creativos y autoconstruidos por las comunidades que los gestionan, aprovechando los saberes de las personas implicadas y adaptando los diseños a las singularidades del entorno, así como minimizando el consumo de recursos. Un urbanismo que contemple de forma simultánea las dimensiones medioambientales, sociales y cognitivas del entorno urbano.

\section{Los huertos comunitarios como cruce de caminos entre el urbanismo participativo y la soberanía alimentaria}

Ante grandes males, muchas soluciones pequeñas, coordinadas, coherentes. M. Max Neef.

Durante las últimas décadas se ha dado un progresivo proceso de acercamiento entre los discursos y las prácticas sobre la ecología urbana, con aquellos relacionados con la democracia y el urbanismo participativo. Las reflexiones sobre la construcción de una ciudad sostenible e inclusiva han comenzado a converger actualmente con los debates sobre la cuestión de la soberanía alimentaria $^{16}$. Un concepto originalmente acuñado por la red Vía Campesina, como aportación crítica a la

sistemas socioecológicos complejos y prácticas de gestión de socioecosistemas (<http://www.stockholmresilience.org/>, consultado el 4 de febrero de 2013)

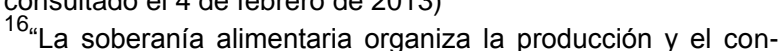
sumo de alimentos en función de las necesidades de las comunidades locales, dando prioridad a la producción para el consumo local. La soberanía alimentaria engloba el derecho a proteger y regular la producción agrícola y ganadera nacional y a proteger el mercado doméstico de entradas de excedentes agrícolas e importaciones de bajo coste de otros países. Las personas sin tierra, los campesinos y los pequeños agricultores deben tener acceso a la tierra, al agua y a las semillas, así como a los recursos productivos y a los servicios públicos. La soberanía y la sostenibilidad alimentarias son una alta prioridad más que las políticas comerciales" Vía Campesina.
Conferencia Mundial sobre la Alimentación que la FAO organizaba en Roma en 1996, donde se enfatizaba la defensa del pequeño productor y la necesidad de que cada nación tenga el derecho de garantizar la alimentación de sus habitantes, respetando la diversidad cultural y productiva (Cuellar y Sevilla, 2009).

La soberanía alimentaria, vista en su complejidad, ofrecería un nuevo paradigma que cuestiona el proceso de globalización económica, la Revolución Verde que ha orientado el desarrollo del actual sistema agroalimentario, la falta de democracia de los mercados y de los procedimientos de decisión política, así como la insostenibilidad ambiental o el patriarcado. Una crítica holística, sustentada en su capacidad para sensibilizar y movilizar a la sociedad civil hacia políticas públicas agrarias integrales, vinculando directamente a los procesos participativos que aspiran a garantizar el acceso a los recursos productivos (tierra, semillas, tecnologías...), introduciendo criterios de sostenibilidad medioambiental y justicia social, enfatizando los mercados locales y los procesos de autonomía que reinventan nuevos derechos individuales y colectivos, en definitiva, esbozando un nuevo modelo de sociedad (Calle et al, 2010). Resulta urgente generalizar las reflexiones sobre el sistema agroalimentario, en el marco de los debates que se están dando sobre la sostenibilidad urbana, ya que las ciudades devienen a medio plazo como los entornos más vulnerables ante previsibles procesos disruptivos como la crisis energética (OECD-IEA, 2011) y el cambio climático (ONU-Habitat, 2011). Modificar los patrones en los que se sustenta el abastecimiento de las ciudades debería ser una variable central en el diseño de cualquier estrategia de transición urbana hacia la sostenibilidad.

Las retóricas de la participación, la sostenibilidad urbana y la soberanía alimentaria, nos hablan de 
recuperar la política y reinventar lo común, de reclamar la inclusión de colectivos y conflictos no representados, así como de repensar la agenda de prioridades del urbanismo convencional.

La traducción a entornos urbanos de las propuestas de soberanía alimentaria nos habla de concebir la ciudad como un espacio desde el que desarrollar procesos de cooperación social, debido a su alta concentración de población y recursos, relocalizando y colectivizando los consumos de productos procedentes de experiencias agroecológicas ${ }^{17}$.

Estos discursos se entrecruzan y superponen de una manera evidente en la reivindicación de lo local como lugar donde se establecen las mayores resonancias y conexiones. Uno de los espacios donde se están dando de forma pionera estos diálogos y desde donde se están tejiendo embrionarias alianzas serían los huertos comunitarios, ya que confluyen iniciativas de microurbanismo participativo con la puesta en marcha de modestas escuelas de soberanía alimentaria. En estos espacios se potencia la sensibilización ciudadana sobre el funcionamiento y los impactos socio-ambientales del sistema agroalimentario, así como la difusión de los análisis y propuestas realizadas desde el entorno social y académico de la Soberanía Alimentaria.

"Vas como a capas, al principio no te planteas lo de la soberanía alimentaria pero sí te planteas comer sano, y después de comer sano qué tiene un impacto menor porque es de una huerta cercana, detrás el tema de las semillas, ya te pones a pensar y llegas" 18

"A la gente que no tiene experiencia y acaba en un huerto urbano, porque se ha puesto de moda, el hecho de probar un tomate de huerta les cambia to-

\footnotetext{
${ }^{17}$ Estas experiencias encierran una elevada pluralidad de formatos que van desde las cooperativas de consumidores con tiendas abiertas al público a grupos de consumo autogestionados, mercados locales o ferias que se realizan periódicamente, venta por Internet...

${ }^{18}$ Entrevista realizada por los autores a miembros Red de Huertos.
}

talmente, es como comerse la pastilla roja de Matrix, que te cambia la percepción de la realidad ${ }^{19,}$ Además estos espacios ponen en valor la actividad agraria en el entorno urbano, sirviendo como nodo para las alianzas campo ciudad, y como puerta de acceso a los principales procesos de cooperación alternativos en cuestiones agroalimentarias (grupos de consumo, circuitos cortos de comercialización...). También favorecen la sostenibilidad ambiental, mediante la inserción de naturaleza en la ciudad, el aumento de áreas verdes, la visibilización de los ciclos estacionales, dando a conocer las variedades locales, o mediante la promoción de hábitos ecológicos, con prácticas como el compostaje de residuos domésticos.

\section{Conclusiones}

Cada frase que pronuncio no debe considerarse una afirmación, sino una pregunta. Neil Bohr.

Hoy por hoy, los huertos comunitarios son principalmente productores de convivencialidad y un recurso de pedagogía política, de forma secundaria producen verduras y hortalizas mientras socializan conocimientos hortícolas. No dan de comer más que de forma testimonial, pero se proyectan hacia el futuro alimentando otros modelos de ciudad y de sistema agroalimentario, se piensan como el antecedente natural de un modelo complejo e integral de agricultura urbana en la que ésta se entiende como una red más de las que conforman el sistema urbano, con espacios diferentes en las distintas escalas: local, urbana y territorial. Una red de espacios que en su conjunto y por su variedad pudiera ser productivamente significativa en lo relativo al abastecimiento, a la vez que cumplir una función demostrativa y educativa, haciendo visible la actividad agrícola mediante un paisaje productivo presente en los distintos espacios de la transición urbano-rural

\footnotetext{
${ }^{19}$ Ibíd.
} 
(cultivos en terrazas, azoteas, jardines, vacíos urbanos, protección de anillos agrícolas periurbanos...), facilitando así el acceso de cualquier persona a los espacios y saberes hortícolas.

En espacios urbanísticamente muy consolidados y con un alto grado de compacidad los huertos comunitarios son la única fórmula factible de facilitar el acceso a tierra cultivable. El hecho de que la organización sea colectiva y comunitaria posibilita intensificar el volumen de gente que participa en relación a la superficie ocupada.

Estas experiencias articulan localmente una pluralidad de sensibilidades, demandas y reivindicaciones (ambientales, vecinales, políticas, relacionales...), a la vez que simultáneamente vienen a plantear la necesaria reterritorialización de la actividad productiva, del ejercicio político de la ciudadanía mediante la intensificación de la defensa del lugar y el territorio. "Lo que importa es que exista un proyecto colectivo enraizado en un territorio como lugar de vida en común y por lo tanto un lugar que debe de preservarse y cuidarse para el bien de todos. La dimensión ya no es un problema topográfico sino social. Se trata del espacio del reconocimiento de la identidad y de la capacidad de acción coordinada y solidaria" (Latouche, 2009).

Lugares ideales desde los que reconstruir el maltrecho lazo social, donde echar raíces ante la inestabilidad y fragilidad de los vínculos en las sociedades actuales (laborales, territoriales, relacionales...). Una forma privilegiada de desarrollar la conciencia de lugar (Magnaghi, 2012) que permita comprender el territorio como espacio de encuentro de una pluralidad de sujetos de cambio precarizados, fragmentados y dispersados geográfica y políticamente. Un encuentro que posibilite la construcción de redes sociales y tejido comunitario desde una perspectiva inclusiva de la pertenencia y la convivencia, así como volver a poner en valor las cualidades particulares del territorio. Todo esto mediante el impulso de inéditas fórmulas de economía social y de nuevas instituciones locales que defiendan la descentralización y el principio de subsidiaridad, que reviertan las dinámicas centro/periferia y promuevan reequilibrios territoriales, mediante la activación del patrimonio colectivo territorial (agroforestal, espacios abiertos, construido e inmaterial) sin comprometer la capacidad de auto-reproducción social y ecológica de la propia comunidad.

Los huertos comunitarios deben ser la palanca sobre la que apoyarse para que la agricultura urbana deje de ser un elemento anecdótico a la hora de diseñar y configurar los asentamientos humanos. La actividad hortícola en la ciudad debe concebirse simultáneamente como un principio, un medio y un fin. Una exigencia de inclusión de estas cuestiones en el planeamiento urbano y la ordenación territorial, una herramienta para conseguirlo y una propuesta política para mejorar la calidad de ciudades, haciéndolas menos vulnerables ante graves desafíos como la crisis energética o el cambio climático.

La proliferación de los huertos comunitarios y su coordinación, pasando de islotes verdes dispersos por la ciudad a un archipiélago interrelacionado, apuntan hacia una forma más sostenible y participativa de concebir la ciudad y la ciudadanía. Muchos espacios reducidos que, como los pequeños habitantes del Lilliput de Los Viajes de Gulliver, son capaces de poner freno y contener al gigante. Una metáfora que ilustra los modestos e imprescindible aportes de iniciativas como estas a la hora de enfrentar el desafío socio-ecológico al que nos abocan las actuales dinámicas de la globalización económica.

\section{Referencias}

ALEXANDER, Christopher et al. Urbanismo $y$ participación. El caso de la Universidad de Ore- 
gón. Barcelona: Editorial Gustavo Gili, 1976.

PUENTE, Raúl. Los huertos urbanos de Sevilla: de la tradición a la novedad. Sevilla: Ed. Diputación de Sevilla, 2012.

AROSAMENA, Graciela. Agricultura urbana. Espacios de cultivo para una ciudad sostenible. Barcelona: Ed. Gustavo Gili, 2012.

BARTHEL, Stephan; FOLKE, Carl; COLDING, Johan. Social-ecological memory in urban gardens-Retaining the capacity for management of ecosystem services. Global Environmental Change, 2010, ํㅡㄴ 20, p. 255-265.

BORJA, Jordi. La ciudad conquistada. Madrid: Ed. Alianza, 2003, 124 p.

CALLE, Ángel; SOLER, Marta y RIVERA, Marta. Soberanía alimentaria y Agroecología Emergente: la democracia alimentaria, en CALLE, Ángel (coord.). Democracias radicales. Barcelona: Ed. Icaria, 2011, p. 213 - 238.

CHOMBART DE LAUWE, Paul Henry. Hombres $y$ ciudades. Barcelona: Ed. Labor, 1976.

CUELLAR, Mamen y SEVILLA, Eduardo. Aportando a la construcción de la soberanía alimentaria desde la agroecología. Revista Ecología Política, 2009 no 38, p. 43-52.

FAO; Agricultura climáticamente inteligente. Políticas, prácticas y financiación para la seguridad alimentaria, adaptación y mitigación. Ed. FAO, 2010.

<http://www.fao.org/docrep/013/i1881s/i1881s00 .pdf> (consultado el 4 de febrero de 2013).

FERNANDEZ, José Luis y RAMOS, Alfredo. Aceras, plazas y parques: la potencialidad de la ecología urbana y las prácticas barriales. Papeles. Revista de relaciones ecosociales y cambio global, 2010, № 111, p. 67-76.

FERNÁNDEZ, José Luis. Huertos comunitarios en Madrid.. Curso Experto Internacional en Soberanía Alimentaria y Agroecología emergente de la Universidad Internacional de Andalucía, 2012. Madrid:

inédito.
$<$ http://portal.ucm.es/c/document_library/get_file ?uuid=746a5010-1eb0-47c2-8315acccc70b2fb7\&groupld=186577> (consultado el 4 de febrero de 2013).

FOLKE, Carl; HAHN, Thomas; OLSSON, Per. Adaptive Governance of Social-Ecological Systems. Annual Review of Environment and Resources, 2005, nํㅜ 30, p. 441-473.

HERNANDO, Alberto. Saul Alinsky: Manual para el agitador para una acción directa noviolenta, Revista El viejo topo, 2006, ㄲo 76.

ILLICH, Ivan. Reivindicar la casa. Diario El País, 5 Junio 1983.

KARANJA, Nancy y NJENGA, Mary. Alimentar las ciudades en VV.AA. La situación del mundo 2011. Ed. Icaria, 2011.

LAWSON, L.; City Bountyful. A century of community gardening in América. Ed. University of California, 2005.

LATOUCHE, Serge. La apuesta por el decrecimiento. Cómo salir del imaginario dominante. Barcelona: Ed Icaria, 2009.

LERNER, Jaime. Acupuntura Urbana. Barcelona: Ed. IACC, 2003.

LINN, Karl. Building Commons and Community. Ed. New Village Press, 2009.

MAGNAGHI, Alberto. El proyecto local. Hacia la conciencia de lugar. Barcelona: Ed. UPC, 2012.

MARTín BARBERO, Jesús. De los medios a las mediaciones. Barcelona: Ed. Gustavo Gili, 1987.

OECD, IEA. World Energy Outlook 2011. París: Ed. Agencia Internacional de la Energía IEA, 2011.

ONU-HABITAT. Las ciudades y el cambio climático: orientaciones para políticas. Río de Janeiro: Ed. Programa de Naciones Unidas para los Asentamientos Humanos, 2011.

SOJA, Edward. Postmetrópolis. Estudios críticos sobre las ciudades y las regiones. Madrid: Ed. Traficantes de Sueños, 2009.

TUAN, Yi-Fu. Topofilia. Ed. Melusina, 2007. 
VERDAGUER, Carlos y VAZQUEZ, Mariano. (Coords) El espacio agrícola entre el campo y la ciudad. Instituto Juan de Herrera. ETSAM, 2010. VIDAL, Tomeu y POL, Enric. La apropiación del espacio una propuesta teórica. Anuario de Psicología, 2005, vol 36, 꾸․ Barcelona.

VV.AA. Informe Ciudades. Hacia un pacto de las ciudades españolas ante el cambio global. Cambio Global España 2020/2050. Madrid: Centro Complutense de Estudios e Información Medioambiental, 2009.

VILLASANTE, Tomás. Desbordes creativos. Estilos y estrategias para la transformación social. Madrid: Ed. Catarata, 2006.

\section{Cita del artículo}

FERNÁNDEZ DE CASADEVANTE, J. L., MORÁN ALONSO, N. Nos plantamos! Urbanismo participativo y agricultura urbana en los huertos comunitarios de Madrid Hábitat y Sociedad, 2012, ํo 4, p. 55-71. $<$ www.habitatysociedad.us.es $>$.

http://dx.doi.org/10.12795/HabitatySociedad.2012.i4.04 